\title{
DIVERSITY OF MICROORGANISMS CAUSING SOFT ROT DISEASE OF FRUITS AND VEGETABLES MARKETED IN TAMANGHASSET (ALGERIA)
}

\author{
Asmaa Benaissa ${ }^{1,2 \bowtie}$. Nadine Chahrazade Djellout ${ }^{2}$. Khadidja Baika ${ }^{2}$. Hafsa Bamebarek ${ }^{2}$, \\ Merdia Bestami ${ }^{2}$ \\ ${ }^{1}$ Laboratory of Plant Physiology, Department of Biology and Physiology of Organisms, Faculty of Biological \\ ${ }^{2}$ Sciences, University of Sciences and Technologies of Houari Boumediene - El-Alia BP 16011 Bab Ezzouar \\ Algiers, Algeria \\ ${ }^{3}$ Department of Biology, Faculty of Sciences and Technology, Universitary of Amine Elokkal ElHadj Moussa \\ Eg. Akhamoukh, 11039 Sersouf, Tamanrasset, Algeria \\ 凶benaissa.asmaa@yahoo.fr \\ https://doi.org/10.34302/crpjfst/2021.13.3.8 \\ Article history: \\ Received: \\ 25 December 2020 \\ Accepted: \\ 2 July 2021 \\ Keywords: \\ Commercial fruit and \\ vegetables; \\ soft rot; \\ microorganisms; \\ PCWDE; \\ Tamanghasset.

\section{ABSTRACT} \\ The nutritional richness of fruit and vegetables makes them an ideal target \\ for microorganisms, causing in particular soft rot. The aim of this work is \\ therefore to isolate, characterize and identify these microorganisms, \\ based on random sampling of rotten fruits and vegetables marketed in the \\ city of Tamanghasset (Algerian Sahara). The study was carried out on 76 \\ samples of fruits and vegetables showing symptoms of soft rot (from 2 to \\ 14 units each). The strains were characterized phenotypically, \\ enzymatically and pathogenically. In addition, the antimicrobial activity of \\ sodium bicarbonates, white vinegar and bleach, was investigated in-vitro \\ on the isolates. The results showed a high diversity of microorganisms \\ involved. The dominance of yeasts was reported, especially of the Candida \\ genus, followed by the Fungi Cladosporium and Botrytis and finally the \\ bacteria which the most important ones are Staphylococcus xylosus and \\ Neisseria cinerae. In addition, these microorganisms are capable to \\ produce a wide range of PCWDE (Plant Cell Wall Degrading Enzymes), \\ represented according to the experimental case by: gelatinase $(30.26 \%)$, \\ caseinase $(31.57 \%)$, amylase $(4.47 \%)$, lipase $(35.52 \%)$, lipoproteinase \\ $(63.15 \%)$ and haemolysin $(30.26 \%)$. However, the production of pectinase \\ in $27.63 \%$ of the isolated microorganisms which is the most \\ phytopathogenic enzyme for the deterioration of fruits and vegetables. This \\ was confirmed by the pathogenicity test on three types of fruits and \\ vegetables (zucchini, mandarin, tomato). Furthermore, the in-vitro \\ evaluation of the sensitivity of these pectinolytic microorganisms to the \\ three usual disinfectants, showed a remarkable efficiency of white vinegar \\ and sodium bicarbonate, but none with bleach. Finally, it is clear from this \\ work that the microorganisms involved in soft rot present a significant \\ taxonomic diversity. Their capacity to resist disinfectants and their \\ enzymatic background are the main factors of pathogenicity.
}

\section{Introduction}

Worldwide, concerns about plant diseases that can affect agriculture are becoming increasingly serious due to severe crop failures, and economic losses (Aouar, 2012). Approximately $30 \%$ of fresh vegetables are lost due to spoilage, primarily due to 
phytopathogenic microorganisms colonization (Ife Fitz and Bas, 2003; Lee et al., 2013). Chemical reactions that cause unpleasant sensory changes in food are the result of microbes' presence that use food as a source of carbon and energy (Gram et al., 2002). Generally, deterioration leads to undesirable changes at organoleptic level, nutritional and healthy quality of food. This type of deterioration known as decay, some of which appear soft, reducing the shelf life of fruits and vegetables (Rosset 1990; Hozbor et al., 2006). However, the main cause of soft rot in fruits and vegetables is the proliferation of bacteria, Fungi and sometimes yeast (Wallen, 1983). These microorganisms use the nutrients (sugar, protein, fat and vitamins) found in the plant product to survive (Ife Fitz and Bas, 2003; Hozbor et al., 2006). However, some opportunistic germs can infect fruits and vegetables already damaged by other phytopathogenic microorganisms that have penetrated the protective wall of vegetables (Lee et al., 2013).

The pathogenicity of these microorganisms is mainly related to the production of a wide range of enzymes called PCWDE (Plant Cell Wall Degrading Enzymes), which have the ability to degrade plant structures composed of: pectins, cellulose, hemicellulose and others, causing cell necrosis and tissue maceration (Lee et al., 2013). Bacterial deterioration first results in tissue softening as pectin degrades (Rawat, 2015). Several bacterial species can penetrate the protective shell of vegetables and damage these products, while others can only penetrate when the product has been damaged (Alfano and Collmer, 1996). Deteriorating bacteria are found on the plant surface and soil and may enter the host from damaged areas or at natural openings during field crop growth, harvest and post-harvest handling, or during storage and distribution (Perombelon, 2002).
To our knowledge, no studies have been carried out on the microorganisms causing soft rot of fruits and vegetables marketed in the arid region of Tamanghasset. This has encouraged us to work in this direction, first know the diversity of the microorganisms involved, in order to test their phytopathogenicity and the production of the main PCWDE related to vegetable deterioration on the one hand, and the usual disinfecting agents on the other hand, to control this disease.

\section{Materials and methods}

\subsection{Materials}

\subsubsection{Sample site and collection}

Seventy-six (76) units of soft rot fruits and vegetables were sampled in February 2020 at various stores (Figure 1) in the city of Tamanghasset (2000 km south of the Algerian coast: Latitude: 22.7869 , Longitude: 5.52722 $22^{\circ} 4713$ North, $5^{\circ} 3138$ East). The samples are presented unpacked in the sampling sites according to their type (each type of crop being in a different stand from the other), and placed in an insulated plastic bag suitable for food use, hermetically sealed and labelled to identify the samples (number, location and date of sampling).

\subsection{Methods}

\subsubsection{Isolation and Purification of} Microorganisms from rotten fruits and vegetables

The damaged plant tissue (object of microbial infection) or the resulting liquid has been spread on the nutrient agar (Laboratory of Conda S.A, Madrid, Spain), as shown in Figure 2. After incubation, the isolates were purified according to the type of microorganism observed under an optical microscope, which led us to use two culture media: (1) PCA (Plate Count Agar) medium (Laboratory of Conda S.A, Madrid, Spain): for the isolation of bacteria and (2) OGA Milieu (Oxytetracycline Gelose Agar) (Institut Pasteur, Algiers, Algeria): for the isolation of yeasts and fungi. 


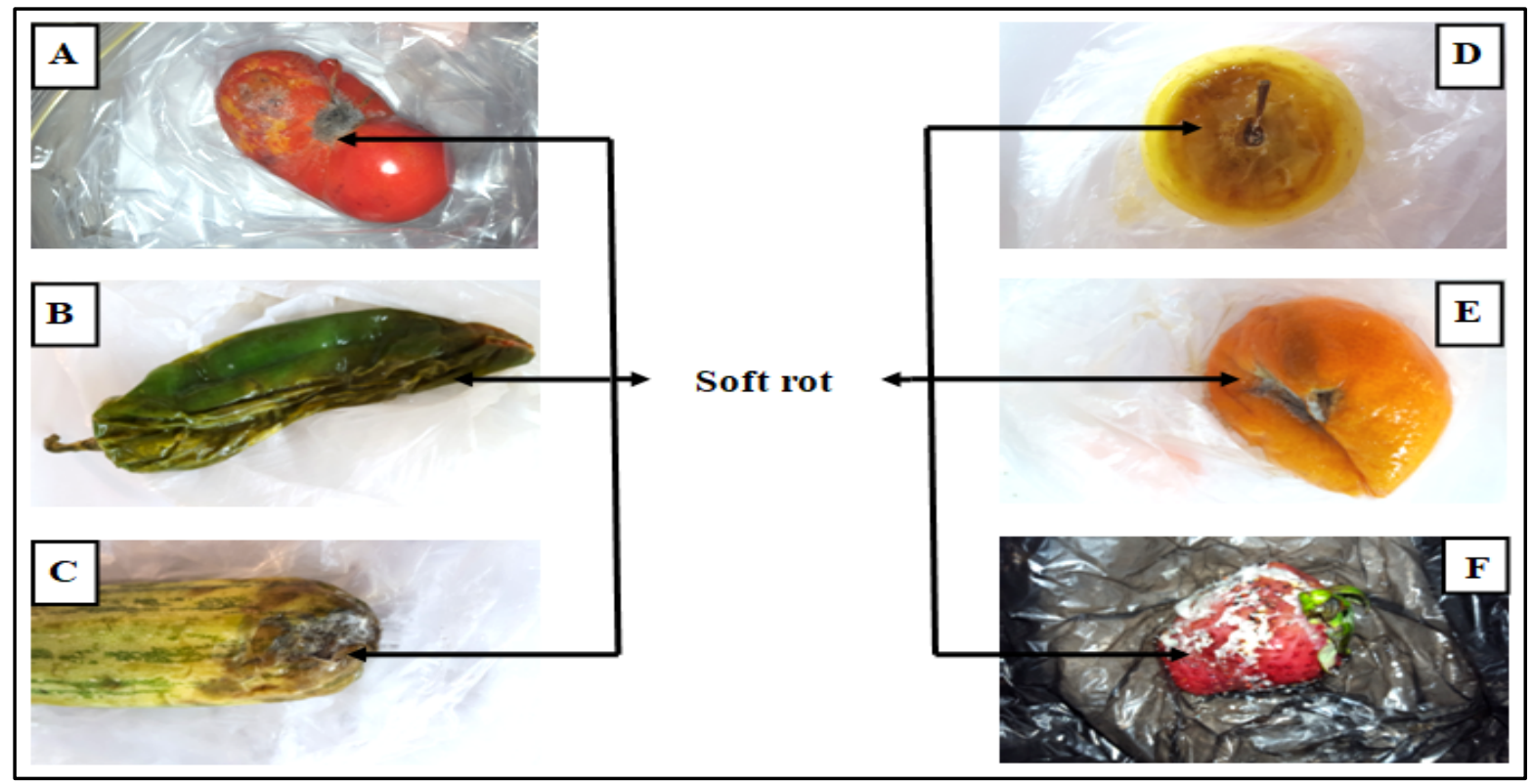

Figure 1. Photograph of some rotten fruits and vegetables sampled in the shops of Tamanghasset city (A- Tomato with white and viscous rot, B- Pepper with brown and viscous rot, C- Zucchini with brown rot with hypha, D-E- Apple and Mandarin with wound in the stem, F- Strawberry with white rot on the surface).

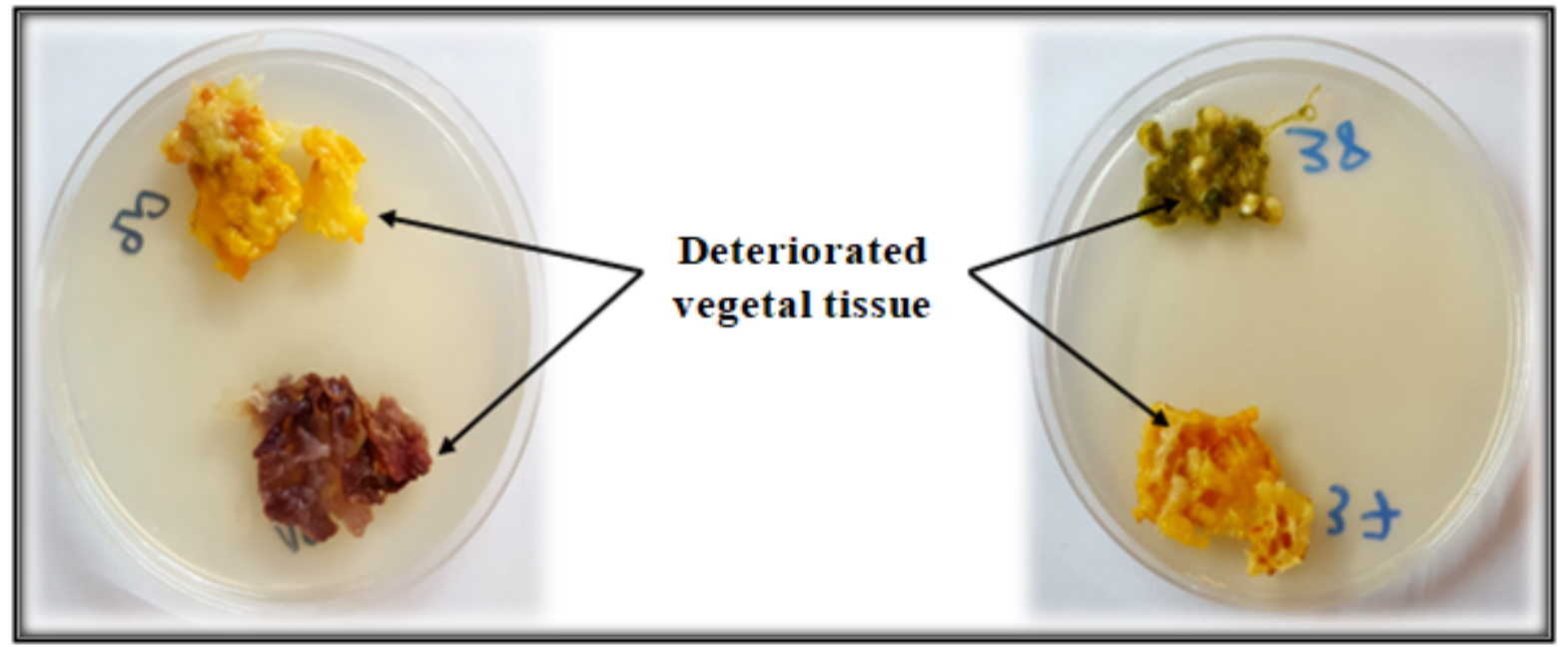

Figure 2. Photograph the isolation of the microorganisms responsible for the soft rot from the damaged tissues before incubation (part of the tissues from the infection site and a few drops of the resulting liquid are spread on the agar).

\subsubsection{Screening for enzymatic activity (PCWDE)}

The enzymatic activity of soft rot microorganisms is related to the presence of a set of enzymes. The hydrolysis test of gelatin was performed as described by Egamberdiyeva
(2004) modified, using a nutrient broth supplemented with $50 \mathrm{~g} / \mathrm{L}$ gelatin powder as a solidifying agent. Casein hydrolysis is tested on Mueller Hinton agar (MH) supplemented with $10 \%$ skimmed milk (CastroEscarpulli et al., 2003) and amylolytic activity was detected on 
Tryptic Soy Agar (TSA 1/10) with 1\% starch added (Delarras 2014). Lecithinase was revealed on an ordinary nutrient agar supplemented by an emulsion of egg yolk and distilled water $(2 \mathrm{~mL} / 20 \mathrm{~mL})$ (Delarras, 2007). Pectinase is sought in a medium consisting of: $15 \mathrm{~g}$ of agar in $519 \mathrm{~mL}$ of distilled water, $1 \mathrm{~g}$ of yeast extract in $20 \mathrm{~mL}$ $\left(\mathrm{NH}_{4}\right)_{2} \mathrm{SO}_{4} \quad 20 \%, 5 \mathrm{~mL}$ of $87 \%$ aqueous glycerol solution, $250 \mathrm{~mL}$ of $2 \%$ polygalacturonic acid aqueous solution, $0.2 \mathrm{M}$ phosphate buffer at $\mathrm{pH}=8,100 \mathrm{~mL}$ distilled water, $1 \mathrm{~mL} \mathrm{MgSO}_{4} 7 \mathrm{H}_{2} \mathrm{O}$ to $1 \mathrm{M}$ (Snaiki et al., 2006). Haemolytic activity of strains has been studied on blood agar (oxoid) and recorded as "positive" when a clear, colorless area around the colonies appeared or "weak" when incomplete hemolysis was observed (Hoeffler, 1977).

\subsubsection{Phenotypical characterization of isolates}

The strains were phenotypically characterized using standard procedures of Gram (C), catalase and oxidase tests supplemented by several biochemical tests as nitrate reductases, sugar assimilation (glucose, fructose, lactose and sucrose), growth at $4^{\circ} \mathrm{C}$ and $42^{\circ} \mathrm{C}$ and growth in salinity condition $(7.5 \% \mathrm{NaCl})$. All strains were stored in a nutrient broth containing $20 \%$ glycerol at $-80^{\circ}$ C. The morphology of isolated microorganisms is studied according to two (02) types of observation: macroscopic and microscopic. However, microscopic observation includes two types of examination: (1) examination in the fresh state and (2) examination after coloring. In addition to the examination in the fresh yeast observation was carried out by adding a few drops of iodine solution (Lugol) (Laboratory of Conda S.A., Madrid, Spain) on the slide in the fresh state. After 2-3 minutes, brown inclusions appear, representing the glycogen seeds present in the yeast cell (Dolisi, 2007). Methylene blue is used to visualize the structure of yeast and Fungi. Pure isolated fungi have been identified in accordance with the recommendations of Dufresne and Guy (2018).
In addition, the identification of bacterial strains was carried out using different types of API galleries: 20 Staph, 20 Strep, $20 \mathrm{NE}$ (BioMérieux, Lyon, France), and their reading is done according to the procedure by the Excel Taxon 2007 software for gallery 20 Strep and Api $\mathrm{Web}^{\mathrm{TM}}$ for gallery $20 \mathrm{NE}$ and $20 \mathrm{Staph}$, in order to have reliable identification results.

\subsubsection{Pathogenicity test}

In order to study the relationship between the pectinolytic strains and soft rot, a test is conducted on intact fruit and vegetables. In fact, we have chosen to apply a procedure below to the intact samples spread at this time of year; namely: zucchini, mandarin and tomato. A disinfection of the surface of the whole fruit and vegetables in alcohol at $70^{\circ}$, and leave for 1 minute, in order to eliminate the saprophytic flora, then rinses the samples for 3 minutes with distilled water. After that, samples are dried in the oven at $30^{\circ} \mathrm{C}$. On the other hand, the suspensions of the pectinolytic strains were sown by swabbing on the fruit and vegetables (three samples for each strain). The results were interpreted with the naked eye, assessing the degree of deterioration (soft rot) by each strain.

\subsubsection{Evaluation of the antimicrobial activity of common liquid disinfectants on pectinolytic microorganisms}

To control the soft rot disease of fruit and vegetable consumption, we used the descriptions provided by the FDA (Sanchez, 2018): [1 tablespoon ( $20 \mathrm{~g}$ ) sodium bicarbonate in $1 \mathrm{~L}$ distilled water; $240 \mathrm{~mL}$ white vinegar in $1 \mathrm{~L}$ distilled water] and FAO (Lopez Camelo, 2007): [1 L domestic bleach diluted in $400 \mathrm{~L}$ distilled water]. For this, the agar direct diffusion technique (Miyadoh, 1993) was used to assess the effect of some common disinfectants on pectinolytic microorganisms, which are considered among the most dangerous plant pathogens.

All values are the mean \pm SE (standard error) of three replicates of a single sample. The obtained data have been submitted to ANOVA 
using the Statistical Analysis System (XLSTAT) version 2016. 02.

\section{Results and discussions}

This work is conducted to study the microorganisms responsible for soft rot disease of fruits and vegetables marketed in the city of Tamanghasset. This study is considered as the first report on the state of this plant disease in the Algerian Sahara. The purpose consists first of isolating these microorganisms and then characterizing them at the morphological, physiological and biochemical levels, then identify and preserve them, in order to launch research to combat them in a future study.

\subsection{Isolated microorganisms responsible for soft rot}

Out of seventy-six (76) isolated strains: thirteen (13) bacteria, forty-nine (49) yeasts and fourteen (14) fungi were found. Figure 3 represents the percentage of each phylum relative to the total number of isolates.

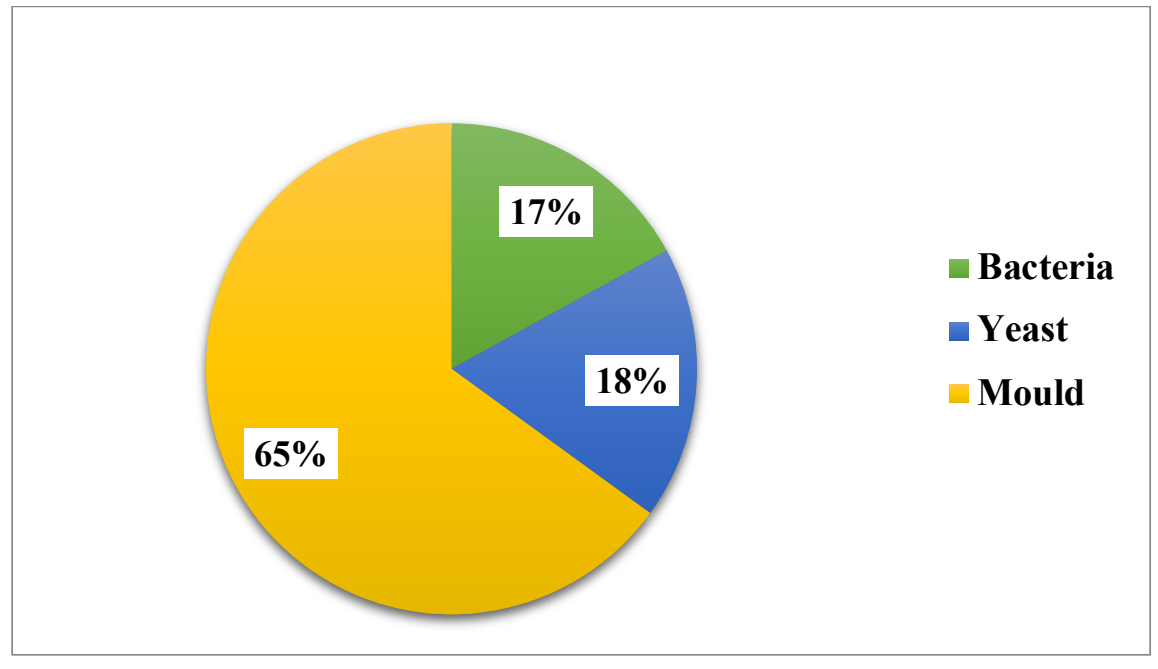

Figure 3. Percentage of each phylum of microorganisms involved in the soft rot of fruits and vegetables marketed in the city of Tamanghasset (southern Algeria).

\subsubsection{Phenotypical characterization of isolated microorganisms}

Characterization of the seventy-six (76) isolated microorganisms (bacteria, yeasts and fungi) was made according to the characteristics described above, the results of which are presented in the following paragraphs.

\subsubsection{Bacteria}

The results of staining (Table 1) showed that among the thirteen (13) bacterial strains found: nine (09) strains are cocci (5 Gram positive and 4 Gram negative) and four (4) strains (one 1 Gram positive and three 3 Grams negative) are rod.

\subsubsection{Yeast and Fungi}

Of the sixty-three (63) isolated yeasts and Fungi, and based on the macroscopic aspect of the colonies, we were able to distinguish two (02) groups: I. Thirty-nine (39) colonies have a smooth appearance, with a creamy or mucous or dry consistency and II. Twenty-three (23) colonies have a rough appearance, with a creamy or dry consistency. In addition, sixteen (16) colonies have aerial hyphae and four (04) colonies have spread green pigment on the agar (Table 2).

The results of the microscopic observation allowed us to visualize two (02) types: yeast and Fungi, where the differentiation between 
them was carried out using an iodine solution, which colors the glycogen seeds existing in the yeast cells with a brown color under the microscope, also the appearance of the cells after staining with methylene blue. We noted that the most yeasts have a pseudo or true mycelium, with spores of the arthrospore or chlamydospore type (Figure 4). Unlike Fungi, observation of the yeasts under a photonic microscope allowed us to visualize the type of budding (bipolar or multilateral) for a few that were probably in the growth stage.

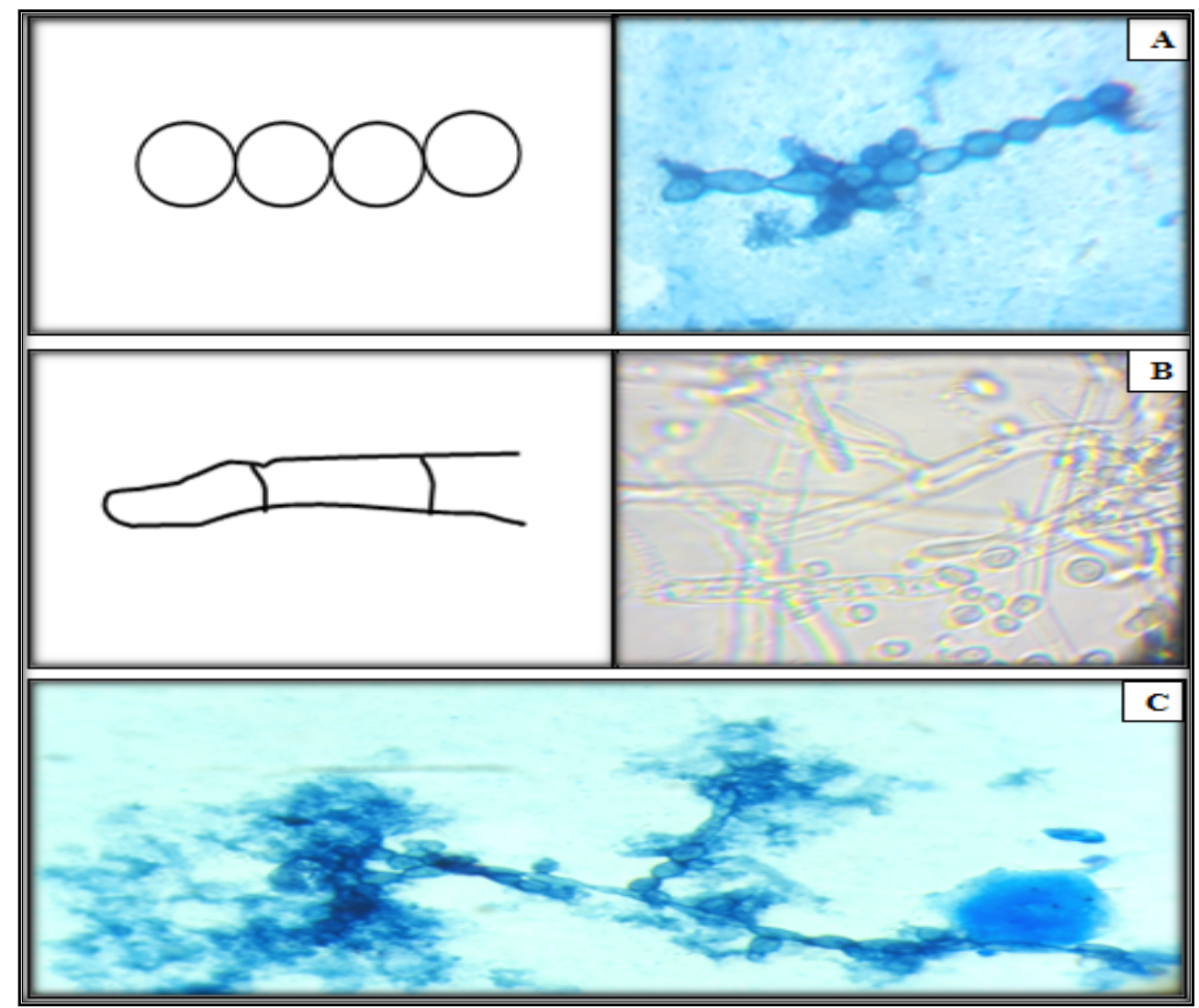

Figure 4. Microscopic observation photograph of yeast and Fungi involved in the soft rot of fruits and vegetables marketed in the city of Tamanghasset (Southern Algeria) under an optical microscope with X40 magnification (B- Yeast with true mycelium, chlamydospore) and X100 magnification (A- Yeast with pseudomycelium, arthrospore; C- Fungi with true mycelium, chlamydospore).

\subsubsection{Physiological characterization}

We have found that of the seventy-six (76) strains isolated, seventy (70) strains possess catalase; most of them are bacteria and yeasts. In addition, we found that among the 76 strains isolated, only thirty-four (34) strains possess cytochrome oxidase, and these strains are mostly yeasts. From the results obtained and presented in tables 1 and 2, we observed that most strains do not possess the enzyme nitrate reductase.
A relative neutral effect of abiotic stress referred to as salinity $(7.5 \% \mathrm{NaCl}$ growth) and temperature $\left(4^{\circ} \mathrm{C}\right.$ and $42^{\circ} \mathrm{C}$ growth $)$ on microbial isolates showed their ability to survive under these conditions (Table 1 and 2). This finding concludes the virulence of our strains, which are principally inhibited by high salinity and low storage temperature (Selmaoui et al., 2016; Benidire et al., 2015). 
Table 1. Phenotypic characterization of isolated bacteria

\begin{tabular}{|c|c|c|c|c|c|c|c|c|c|c|c|c|}
\hline \multirow{3}{*}{ 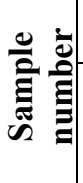 } & \multicolumn{4}{|c|}{ Microscopic aspect } & \multicolumn{6}{|c|}{ Physiological characters } & \multirow{3}{*}{$\begin{array}{l}\text { Genus and } \\
\text { species }\end{array}$} & \multirow{3}{*}{ Vegetal host } \\
\hline & \multirow{2}{*}{ Form } & \multirow{2}{*}{$\begin{array}{l}\text { Regroupement } \\
\text { mode }\end{array}$} & \multirow{2}{*}{ Gram } & \multirow{2}{*}{ Mobility } & \multirow{2}{*}{ Catalase } & \multirow{2}{*}{ Oxydase } & \multirow{2}{*}{ NR } & \multirow{2}{*}{$\begin{array}{c}\text { Salinity } \\
7.5 \% \\
\mathrm{NaCl}\end{array}$} & \multicolumn{2}{|c|}{ Temperature } & & \\
\hline & & & & & & & & & $4^{\circ} \mathrm{C}$ & $42{ }^{\circ} \mathrm{C}$ & & \\
\hline 05 & Cocci & Chain & + & + & + & + & - & - & + & + & $\begin{array}{c}\text { Staphylococcus } \\
\text { xylosus }\end{array}$ & Tomato \\
\hline 06 & Cocci & Clusters & - & - & + & - & + & - & + & + & Neisseria cinerea & Orange \\
\hline 21 & Cocci & Chain & + & - & + & + & + & - & + & + & & Strawberry \\
\hline 24 & Cocci & Diplo & + & - & + & - & - & + & + & + & $\begin{array}{c}\text { Staphylococcus } \\
\text { xylosus }\end{array}$ & Strawberry \\
\hline 37 & Cocci & Chain & + & + & + & + & - & - & + & + & $\begin{array}{c}\text { Staphylococcus } \\
\text { xylosus }\end{array}$ & Strawberry \\
\hline 43 & Cocci & $\begin{array}{l}\text { Chain , } \\
\text { isolated }\end{array}$ & + & + & + & - & + & - & + & + & Neisseria mucosa & Mandarin \\
\hline 44 & Cocci & Chain & - & - & + & + & + & - & + & + & Neisseria mисоsa & Mandarin \\
\hline 51 & Cocci & Chain & - & + & + & + & - & + & + & + & Neisseria cinerea & Mandarin \\
\hline 56 & Bacilli & Diplo, isolated & - & + & + & + & - & + & + & + & Vibrio vulnificus & Apple \\
\hline 61 & Bacilli & Diplo & + & + & + & + & + & + & + & + & Bacillus coagulans & Apple \\
\hline 65 & Cocci & Diplo, isolated & - & + & + & + & + & + & + & + & $\begin{array}{c}\text { Haemophilus } \\
\text { paraphraphilus }\end{array}$ & Onion \\
\hline 69 & Bacilli & Diplo, isolated & - & + & + & + & + & + & + & + & $\begin{array}{c}\text { Photobacterium } \\
\text { damselae }\end{array}$ & Onion \\
\hline
\end{tabular}

Gram type: Gram positive (+), Gram négative (-) ;

Mobility: absence (-), presence $(+)$.

Catalase : absence (-), presence $(+)$;

Growth at $4{ }^{\circ} \mathrm{C} / 42{ }^{\circ} \mathrm{C}$ : absence (-), presence.
Oxydase : absence $(-)$, presence $(+)$;

NR (Nitrate réductase) : absence (-), présence $(+)$;

Salinity $7.5 \%$ NaCl : absence (-), presence $(+)$; 
Table 2. Phenotypic characterization of isolated fungi

\begin{tabular}{|c|c|c|c|c|c|c|c|c|c|c|c|c|c|c|}
\hline \multirow{3}{*}{ 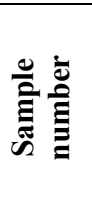 } & \multicolumn{3}{|c|}{ The thallus aspect } & \multicolumn{2}{|c|}{$\begin{array}{c}\text { Microscopic aspect } \\
\text { (hypha) }\end{array}$} & \multirow{3}{*}{$\begin{array}{l}\text { Nature of } \\
\text { the strands }\end{array}$} & \multicolumn{6}{|c|}{ Physiological characters } & \multirow[t]{3}{*}{ Genus and species } & \multirow[t]{3}{*}{ Vegetal host } \\
\hline & \multirow{2}{*}{$\begin{array}{c}\text { Aerial } \\
\text { mycelium }\end{array}$} & \multirow{2}{*}{ Color } & \multirow{2}{*}{$\begin{array}{c}\text { Diffusible } \\
\text { pigment }\end{array}$} & \multirow{2}{*}{$\begin{array}{c}\text { Type of } \\
\text { mycelium }\end{array}$} & \multirow{2}{*}{ spores } & & \multirow{2}{*}{ Catalase } & \multirow{2}{*}{ Oxydase } & \multirow{2}{*}{ NR } & \multirow{2}{*}{$\begin{array}{c}\text { Salinity } \\
7.5 \% \\
\mathrm{NaCl} \\
\end{array}$} & \multicolumn{2}{|c|}{ Temperature } & & \\
\hline & & & & & & & & & & & $4^{\circ} \mathrm{C}$ & $4^{\circ} \mathrm{C}$ & & \\
\hline 1 & - & Yellow & - & TM & $\mathrm{C}$ & Fungus & + & - & - & - & + & + & Candida kefyr & Mandarin \\
\hline 2 & - & Yellow & - & TM & $\mathbf{A}$ & Fungus & + & - & - & + & + & + & Geotrichum capitatum & Mandarin \\
\hline 3 & - & White & - & $\mathbf{P M}$ & $\mathbf{A}$ & Fungus & + & + & - & + & + & + & Pichia anomala & Mandarin \\
\hline 4 & - & White & - & TM & $\mathbf{A}$ & Fungus & + & + & - & - & + & + & Trichosporon $s p$ & Zucchini \\
\hline 7 & - & White & - & TM & $\mathbf{A}$ & Fungus & + & - & - & - & + & & Candida guilliermondii & Tomato \\
\hline 8 & + & Yellow & - & TM & C & Fungus & - & + & - & - & + & + & Candida kefyr & Tomato \\
\hline 9 & - & Jaune & - & $\mathbf{P M}$ & $\mathbf{C}$ & Fungus & + & - & - & + & + & + & Candida kefyr & Apple \\
\hline 10 & - & White & - & $\begin{array}{c}\text { TM, } \\
\text { septate }\end{array}$ & $\mathrm{C}$ & Fungus & + & - & + & + & + & + & Candida tropicalis & Tomato \\
\hline 11 & + & White & - & PM & $\mathbf{A}$ & Fungus & + & - & - & + & + & + & Candida dubliensis & Tomato \\
\hline 12 & + & White & - & TM & $\mathbf{A}$ & Fungus & + & - & - & + & + & + & Trichosporon $s p$ & Tomato \\
\hline 13 & + & White & - & TM & $\mathbf{A}$ & Fungus & + & + & + & + & + & + & Candida kefyr & Tomato \\
\hline 14 & - & White & - & TM & $\mathbf{A}$ & Fungus & + & + & + & - & + & + & Candida kefyr & Pepper \\
\hline 15 & - & White & - & $\begin{array}{l}\text { TM, no } \\
\text { septate }\end{array}$ & $\mathbf{A}$ & Fungus & + & - & - & + & + & + & Candida dubliensis & Pepper \\
\hline 16 & + & White & - & TM & $\mathbf{A}$ & Fungus & + & + & - & - & + & + & Candida tropicalis & Zucchini \\
\hline 17 & + & Yellow & - & TM & $\mathbf{A}$ & Fungus & + & - & - & - & + & + & Trichosporon $s p$ & Onion \\
\hline 18 & - & Yellow & + & TM & $\mathrm{C}$ & Fungus & + & + & - & - & + & + & Botrytis $s p$ & Onion \\
\hline 19 & - & White & + & TM & $\mathbf{A}$ & Fungus & + & + & - & - & + & + & Trichosporon $s p$ & Onion \\
\hline 20 & - & White & - & PM & $\mathbf{A}$ & Fungus & + & + & - & + & + & + & Candida guilliermondi & Mandarin \\
\hline 22 & - & White & - & TM & $\mathbf{A}$ & Fungus & - & + & + & + & + & + & Botrytis $s p$ & Orange \\
\hline 23 & - & White & - & TM & $\mathbf{A}$ & Fungus & + & + & - & - & + & + & Botrytis $s p$ & Orange \\
\hline 25 & - & Yellow & - & TM & $\mathbf{C}$ & Fungus & + & + & - & - & + & + & Trichosporon $s p$ & Tomato \\
\hline 26 & - & White & - & PM & $\mathbf{C}$ & Fungus & + & - & - & + & + & + & Candida kefyr & Pepper \\
\hline 27 & - & White & + & $\begin{array}{c}\text { TM, } \\
\text { septate }\end{array}$ & $\mathbf{C}$ & Fungus & + & + & + & - & + & + & Trichosporon $s p$ & Tomato \\
\hline 28 & - & Yellow & - & PM & $\mathbf{A}$ & Fungus & + & - & - & - & + & + & Botrytis cinerae & Zucchini \\
\hline 29 & - & White & - & TM & A & Fungus & + & + & - & + & + & + & Botrytis $s p$ & Onion \\
\hline 30 & - & White & + & TM & $\mathbf{A}$ & Fungus & + & - & - & + & + & + & Trichosporon $s p$ & Pepper \\
\hline
\end{tabular}


Benaissa et al/ Carpathian Journal of Food Science and Technology, 2021, 13(3), 98-115

\begin{tabular}{|c|c|c|c|c|c|c|c|c|c|c|c|c|c|c|}
\hline 31 & - & White & - & TM & $\mathbf{A}$ & Fungus & + & + & - & + & + & + & Trichosporon $s p$ & Pepper \\
\hline 32 & + & White & - & $\begin{array}{l}\text { TM, no } \\
\text { septate }\end{array}$ & $\mathbf{A}$ & Fungus & + & - & - & - & + & + & Trichosporon $s p$ & Pepper \\
\hline 34 & - & White & - & TM & $\mathbf{A}$ & Fungus & + & + & - & + & + & + & Candida dubliensis & Pepper \\
\hline 35 & + & White & - & TM & $\mathbf{A}$ & Fungus & + & - & - & + & + & + & Trichosporon $s p$ & Apple \\
\hline 36 & - & Yellow & - & TM & $\mathbf{A}$ & Fungus & + & + & - & + & + & + & Trichosporon $s p$ & Apple \\
\hline 38 & - & White & - & TM & $\mathbf{A}$ & Fungus & - & - & + & - & - & + & Candida dubliensis & Pepper \\
\hline 39 & - & White & - & PM & $\mathbf{A}$ & Fungus & + & + & - & + & + & + & Pichia anomala & Pepper \\
\hline 40 & - & White & - & PM & $\mathrm{C}$ & Fungus & + & - & + & + & + & + & Candida kefyr & Mandarin \\
\hline 41 & - & White & - & $\mathbf{P M}$ & $\mathbf{C}$ & Fungus & + & - & - & + & + & + & Candida parapsilosis & Eggplant \\
\hline 42 & - & White & - & $\mathbf{P M}$ & $\mathbf{C}$ & Fungus & + & - & + & + & + & + & Pichia anomala & Apple \\
\hline 45 & - & White & - & PM & $\mathbf{A}$ & Fungus & + & - & - & + & + & + & Candida guilliermondii & Mandarin \\
\hline 46 & + & White & - & TM & $\mathbf{A}$ & Fungus & + & - & - & - & + & + & Cladosporium $s p$ & Mandarin \\
\hline 47 & - & White & - & PM & $\mathbf{A}$ & Fungus & + & - & + & - & + & + & Candida kefyr & Mandarin \\
\hline 48 & - & White & - & TM & $\mathbf{A}$ & Fungus & + & - & + & - & + & + & Trichosporon $s p$ & Mandarin \\
\hline 49 & + & White & - & TM & $\mathrm{C}$ & Fungus & + & - & - & - & + & + & Trichospron sp & Mandarin \\
\hline 50 & + & White & - & PM & $\mathbf{C}$ & Fungus & + & - & - & + & + & + & Penicillium $s p$ & Mandarin \\
\hline 52 & - & White & - & $\mathbf{P M}$ & $\mathbf{A}$ & Fungus & + & + & - & + & + & + & Candida guilliermondii & Apple \\
\hline 53 & + & White & - & TM & $\mathbf{A}$ & Fungus & + & - & - & + & + & + & Geotrichum capitatum & Apple \\
\hline 54 & - & White & - & PM & $\mathrm{C}$ & Fungus & + & - & - & + & + & + & Candida kefyr & Apple \\
\hline 55 & - & White & - & PM & $\mathbf{A}$ & Fungus & + & - & - & + & + & + & Cladosporium $s p$ & Apple \\
\hline 57 & - & White & - & PM & $\mathbf{C}$ & Fungus & + & + & + & + & + & + & Geotrichum capitatum & Apple \\
\hline 58 & + & White & - & TM & $\mathbf{A}$ & Fungus & + & - & - & + & + & + & Candida guilliermondii & Apple \\
\hline 59 & - & White & - & TM & $\mathbf{A}$ & Fungus & + & - & - & + & + & + & Cladosporium $s p$ & Apple \\
\hline 60 & - & Yellow & - & TM & $\mathbf{C}$ & Fungus & + & + & + & + & + & + & Fusarium $s p$ & Apple \\
\hline 62 & - & White & - & TM & $\mathrm{C}$ & Fungus & + & - & - & + & + & + & Trichosporon $s p$ & Tomato \\
\hline 63 & - & White & - & TM & $\mathbf{A}$ & Fungus & + & + & - & + & + & + & Penicillium digitatum & Tomato \\
\hline 64 & - & White & - & TM & $\mathbf{A}$ & Fungus & + & + & - & + & + & + & Cladosporium $s p$ & Mandarin \\
\hline 71 & - & White & - & PM & $\mathbf{C}$ & Fungus & + & - & + & + & + & + & Candida kefyr & Tomato \\
\hline 72 & - & White & - & $\mathbf{P M}$ & $\mathbf{A}$ & Fungus & + & + & + & + & + & + & Candida kefyr & Zucchini \\
\hline 73 & - & Yellow & - & TM & $\mathbf{A}$ & Fungus & + & + & - & + & + & + & Botrytis $s p$ & Onion \\
\hline 74 & - & White & - & TM & $\mathbf{A}$ & Fungus & + & - & + & + & + & + & Tricosporon $s p$ & Zucchini \\
\hline 75 & - & White & - & $\mathbf{P M}$ & $\mathbf{A}$ & Fungus & - & - & + & - & + & + & Candida kefyr & Spinach \\
\hline 76 & - & White & - & TM & $\mathrm{C}$ & Fungus & - & - & - & + & + & + & Cladosporium $s p$ & Spinach \\
\hline 77 & - & White & - & TM & $\mathrm{C}$ & Fungus & + & - & + & + & + & + & Trichosporon $s p$ & Wild truffle \\
\hline 78 & + & White & - & PM & $\mathbf{A}$ & Fungus & - & - & - & - & + & + & Penicillium $s p$ & Wild truffle \\
\hline
\end{tabular}




\begin{tabular}{|c|c|c|c|c|c|c|c|c|c|c|c|c|c|c|}
\hline 79 & + & White & - & $\mathbf{P M}$ & $\mathbf{A}$ & Fungus & + & - & + & + & + & + & Candida lypolytica & Wild truffle \\
\hline 80 & + & White & - & TM & $\mathbf{A}$ & Fungus & + & - & + & + & + & + & Geotrichum capitatum & Eggplant \\
\hline
\end{tabular}

Aerial mycelium : absence (-), presence $(+)$;

Diffusible pigment : absence $(-)$, presence $(+)$;

Type of spores: Arthrospore (A), Chlamydospore (C).

Type of mycelium : Pseudomycelium (PM), True mycelium (TM) ;
Catalase : absence $(-)$, presence $(+)$;

Oxydase : absence $(-)$, presence $(+)$;

NR (Nitrate réductase) : absence $(-)$, présence $(+)$;

Growth at $4^{\circ} \mathrm{C} / 42{ }^{\circ} \mathrm{C}$ : absence $(-)$, presence $(+)$.

Table 3. Assessment of the Degree of Soft Rot Caused by Pectinolytic Strains in Three (03) Plant Varieties

\begin{tabular}{|c|c|c|c|c|c|c|c|c|c|c|c|c|c|c|c|c|c|c|c|c|c|c|}
\hline \multirow{2}{*}{\multicolumn{2}{|c|}{$\frac{\text { Strand }}{\text { Code }}$}} & 01 & 04 & 05 & 06 & 11 & 16 & 19 & 20 & 21 & 24 & 25 & 26 & 27 & 28 & 32 & 39 & 40 & 41 & 57 & 62 & 63 \\
\hline & & PS1 & PS2 & PS3 & PS4 & PS5 & PS6 & PS7 & PS8 & PS9 & PS10 & PS11 & PS12 & PS13 & PS14 & PS15 & PS16 & PS17 & PS18 & PS19 & PS20 & PS21 \\
\hline \multirow{2}{*}{ 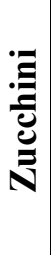 } & 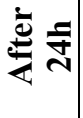 & $\begin{array}{l}+ \\
+\end{array}$ & + & $\begin{array}{l}+ \\
+\end{array}$ & + & ' & + & + & ' & + & + & + & + & $\stackrel{+}{+}$ & + & ' & ' & ' & ' & + & ' & ' \\
\hline & 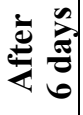 & $\begin{array}{l}+ \\
+\end{array}$ & + & + & $\begin{array}{l}+ \\
+\end{array}$ & + & + & $\stackrel{+}{+}$ & ' & + & + & + & $\stackrel{+}{+}$ & $\stackrel{+}{+}$ & + & ' & I & ' & + & $\stackrel{+}{+}$ & ' & ' \\
\hline \multirow{2}{*}{ 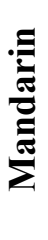 } & 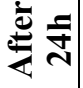 & ' & + & + & I & ' & + & ' & ' & + & + & 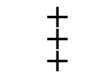 & + & I & I & I & + & + & $\neq$ & + & I & + \\
\hline & 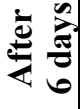 & ' & $\stackrel{+}{+}$ & + & + & + & + & ' & ' & + & + & + & $\stackrel{+}{+}$ & + & + & I & $\begin{array}{l}+ \\
+\end{array}$ & $\begin{array}{l}+ \\
+\end{array}$ & $\stackrel{+}{+}$ & + & + & + \\
\hline \multirow{2}{*}{$\stackrel{\stackrel{\theta}{\tilde{g}}}{\stackrel{\Xi}{0}}$} & $\stackrel{\frac{e}{2}}{\frac{5}{4}}$ & 1 & + & + & I & ' & 1 & + & I & + & I & $\mp$ & 1 & $\dot{+}$ & I & + & I & $\mp$ & I & $\mp$ & + & ' \\
\hline & 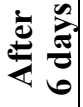 & + & + & + & + & + & 1 & \pm & + & + & + & \pm & \pm & \pm & + & + & + & + & + & \pm & + & ' \\
\hline
\end{tabular}

(-) : No rot ; (+) : Weak rot ; (++) : Medium rot ; (+++) : Strong rot ; (++++) : Very strong rot (appearance of mashed potatoes) ; PS : pectinolytic strand; PS1 : Candida kefyr; PS2 : Trichosporon sp ; PS3 : Staphylococcus xylosus ; PS4 : Neisseria cinerea; PS5 : Candida dubliniensis; PS6 : Candida tropicalis ; PS7 : Trichosporon sp ; PS8 : Candida guilliermondii ; PS9 : Staphylococcus xylosus ; PS10 : Staphylococcus xylosus ; PS11 : Trichosporon sp ; PS12 : Candida kefyr ; PS13 : Trichosporon sp ; PS14 : Botrytis cinerea ; PS15 : Trichosporon sp ; PS16 : Pichia anomala ; SP17 : Candida kefyr ; PS18 : Candida parapsilosis ; PS19 : Geotrichum capitatum ; PS20 : Trichosporon sp ; PS21 : Penicillium digitatum. 
Table 4. Antimicrobial activity of common disinfectants on isolated pectinolytic strains expressed by diameter inhibition zones (mm)

\begin{tabular}{|c|c|c|c|c|c|c|c|c|c|c|c|c|c|c|c|c|c|c|c|c|c|}
\hline Strains & 01 & 04 & 05 & 06 & 11 & 16 & 19 & 20 & 21 & 24 & 25 & 26 & 27 & 28 & 32 & 39 & 40 & 41 & 57 & 62 & 63 \\
\hline Code & PS1 & PS2 & PS3 & PS4 & PS5 & PS6 & PS7 & PS8 & PS9 & PS10 & PS11 & PS12 & PS13 & PS14 & PS15 & PS16 & PS17 & PS18 & PS19 & PS20 & PS21 \\
\hline 吾 & $\begin{array}{c}7 \pm \\
1.08 \mathrm{~b}\end{array}$ & $\begin{array}{c}8.66 \\
\pm \\
1.08 \mathrm{~b}\end{array}$ & $\begin{array}{c}7.66 \pm \\
1.08 \mathrm{~b}\end{array}$ & $\begin{array}{c}8.66 \\
\pm \\
1.08 \mathrm{~b}\end{array}$ & $\begin{array}{c}8 \pm \\
1.08 \mathrm{~b}\end{array}$ & $\begin{array}{c}6.33 \pm \\
1.08 \mathrm{~b}\end{array}$ & $\begin{array}{c}8 \pm \\
1.08 \mathrm{~b}\end{array}$ & $\begin{array}{c}16 \pm \\
1.08 \mathrm{a}\end{array}$ & $\begin{array}{c}8 \pm \\
1.08 \mathrm{~b}\end{array}$ & $\begin{array}{c}0 \pm \\
1.08 \mathrm{c}\end{array}$ & $\begin{array}{c}7 \pm \\
1.08 \mathrm{~b}\end{array}$ & $\begin{array}{c}6 \pm \\
1.08 \mathrm{~b}\end{array}$ & $\begin{array}{l}7.33 \pm \\
1.08 \mathrm{~b}\end{array}$ & $\begin{array}{c}10 \pm \\
1.08 \mathrm{~b}\end{array}$ & $\begin{array}{c}6.66 \\
\pm \\
1.08 \mathrm{~b}\end{array}$ & $\begin{array}{c}8.66 \pm \\
1.08 \mathrm{~b}\end{array}$ & $\begin{array}{c}6.66 \\
\pm \\
1.08 \mathrm{~b}\end{array}$ & $\begin{array}{c}8.33 \pm \\
1.08 \mathrm{~b}\end{array}$ & $\begin{array}{c}9.33 \\
\pm \\
1.08 \mathrm{~b}\end{array}$ & $\begin{array}{c}7 \pm \\
1.08 \mathrm{~b}\end{array}$ & $\begin{array}{c}6.66 \pm \\
1.08 \mathrm{~b}\end{array}$ \\
\hline & $\begin{array}{c}7.66 \\
\pm \\
0.81 \mathrm{a}\end{array}$ & $\begin{array}{c}0 \pm \\
0.81 \mathrm{~b}\end{array}$ & $\begin{array}{c}9.33 \pm \\
0.81 \mathrm{a}\end{array}$ & $\begin{array}{c}7.33 \\
\pm \\
0.81 \mathrm{a}\end{array}$ & $\begin{array}{c}7 \pm \\
0.81 \mathrm{a}\end{array}$ & $\begin{array}{c}9.66 \pm \\
0.81 \mathrm{a}\end{array}$ & $\begin{array}{c}8.33 \pm \\
0.81 \mathrm{a}\end{array}$ & $\begin{array}{c}0 \pm \\
0.81 \mathrm{~b}\end{array}$ & $\begin{array}{c}9.66 \pm \\
0.81 \mathrm{a}\end{array}$ & $\begin{array}{c}8 \pm \\
0.81 \mathrm{a}\end{array}$ & $\begin{array}{c}6.66 \pm \\
0.81 \mathrm{a}\end{array}$ & $\begin{array}{c}6.66 \\
\pm \\
0.81 \mathrm{a}\end{array}$ & $\begin{array}{c}6.33 \pm \\
0.81 \mathrm{a}\end{array}$ & $\begin{array}{c}9.66 \\
\pm \\
0.81 \mathrm{a}\end{array}$ & $\begin{array}{c}9.33 \\
\pm \\
0.81 \mathrm{a}\end{array}$ & $\begin{array}{c}6 \pm \\
0.81 \mathrm{a}\end{array}$ & $\begin{array}{c}6.66 \\
\pm \\
0.81 \mathrm{a}\end{array}$ & $\begin{array}{c}9.33 \pm \\
0.81 \mathrm{a}\end{array}$ & $\begin{array}{c}9.66 \\
\pm \\
0.81 \mathrm{a}\end{array}$ & $\begin{array}{c}6.66 \pm \\
0.81 \mathrm{a}\end{array}$ & $\begin{array}{c}7 \pm \\
0.81 \mathrm{a}\end{array}$ \\
\hline 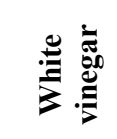 & $\begin{array}{c}10.66 \\
\pm \\
0.83 \mathrm{c}\end{array}$ & $\begin{array}{c}10.33 \\
\pm \\
0.83 \mathrm{c}\end{array}$ & $\begin{array}{l}12.66 \pm \\
0.83 \mathrm{abc}\end{array}$ & $\begin{array}{c}9.66 \\
\pm \\
0.83\end{array}$ & $\begin{array}{c}7.33 \\
\pm \\
0.83\end{array}$ & $\begin{array}{l}12.33 \pm \\
0.83 \mathrm{abc}\end{array}$ & $\begin{array}{c}11.33 \\
\pm \\
0.83 \mathrm{bc}\end{array}$ & $\begin{array}{c}7.33 \\
\pm \\
0.83\end{array}$ & $\begin{array}{c}12 \pm \\
0.83 \mathrm{abc}\end{array}$ & $\begin{array}{l}13.66 \pm \\
0.83 \mathrm{abc}\end{array}$ & $\begin{array}{c}11.33 \\
\pm \\
0.83 \mathrm{bc}\end{array}$ & $\begin{array}{c}0 \pm \\
0.83\end{array}$ & $\begin{array}{l}14.66 \pm \\
0.83 \mathrm{abc}\end{array}$ & $\begin{array}{c}11 \pm \\
0.83 b\end{array}$ & $\begin{array}{c}16 \pm \\
0.83 \mathrm{a}\end{array}$ & $\begin{array}{c}13 \pm \\
0.83 \mathrm{abc}\end{array}$ & $\begin{array}{c}0 \pm \\
0.83\end{array}$ & $\begin{array}{l}13.66 \pm \\
0.83 \mathrm{abc}\end{array}$ & $\begin{array}{l}10 \pm \\
0.83\end{array}$ & $\begin{array}{c}15.66 \\
\pm \\
0.83 \mathrm{ab}\end{array}$ & $\begin{array}{c}15.66 \\
\pm \\
0.83 \mathrm{ab}\end{array}$ \\
\hline
\end{tabular}

PS1 : Candida kefyr ; PS2 : Trichosporon sp ; PS3 : Staphylococcus xylosus ; PS4 : Neisseria cinerea ; PS5 : Candida dubliniensis; PS6 : Candida tropicalis ; PS7 : Trichosporon sp ; PS8 : Candida guilliermondii ; PS9 : Staphylococcus xylosus ; PS10 : Staphylococcus xylosus ; PS11 : Trichosporon sp ; PS12 : Candida kefyr ; PS13 : Trichosporon sp ; PS14 : Botrytis cinerea ; PS15 : Trichosporon sp ; PS16 : Pichia anomala ; SP17 : Candida kefyr ; PS18 : Candida parapsilosis ; PS19 : Geotrichum capitatum ; PS20 : Trichosporon sp ; PS21 : Penicillium digitatum. 
We can explain the survival of these pathogens by their adaptations or their ability to sporulate, which allow them on the one hand, sporulant bacteria (Bacillus) to resist adverse conditions, and on the other hand fungi to propagate and colonize other niches (Pozzi, 2014). Moreover, the city of Tamanghasset in terms of agricultural self-sufficiency is less developed, as it is considered an arid zone, which requires the import of fruits and vegetables from other areas of the country. This transport can probably play a major role in the spread of spores as agents of resistance and propagation of the microorganisms causing soft rot.

\subsubsection{Biochemical Characterization}

\subsubsection{Sugar assimilation}

As part of the characterization of our strains, several phenotypic tests were performed including the assimilation of sugars. We noted a strong assimilation for the three sugars: sucrose, fructose and lactose, compared to glucose (Figure 5). Gupta et al. (2015) explained this latter as the fact that plant pathogens primarily target complex compounds in the early phase of infection, which explains the low metabolization of glucose compared to other sugars. In addition, the assimilation of lactose, which is not part of the carbohydrate composition of fruits and vegetables (Rémésy, 2008), according to Rosset (1995), indicates that these microorganisms are capable to alter other foods.

\subsubsection{Enzymatic activity}

In this same perspective, we studied the enzymatic potential of isolated strains that seem to be a very important player in the alteration of fruits and vegetables. Phytopathogenic microorganisms are capable to produce one or more enzymes, which enable them to break down fruit and vegetable tissues. These enzymes are called PCWDE (Plant Cell Wall Degrading Enzymes) (Lee et al., 2013). We observed that all isolated strains possess this hydrolytic activity, but in different proportions for the selected substrates, the most important of which is lipoprotein-bound, followed by casein and gelatin. Based on the results obtained and presented in Figure 6, all isolated strains have a low capacity for protein hydrolysis (casein and gelatin). However, their hydrolytic properties are notable for lipids, particularly lipoproteins, and for polysaccharides, specifically starch. On the other hand, most strains are not able to hydrolyze hemoglobin (hemolytic activity), and those that have this activity are mainly of the $\beta$ type. This can be explained first, that the majority of our isolates are fungi, whose infections are characterized by the secretion of the protease enzyme in the early stage of infection (Movahedi and Heale, 1990; Zalewsky-Sobcazak, 1985). In addition, our isolates are capable to hydrolyze lipids and polysaccharides (starch), suggesting that they use both substrates as sources of carbon and energy, according to Boiron (1996) and Nicklin et al. (2000). Moreover, some of our phytopathogens have an enzymatic characteristic and not the least, namely the hydrolysis of pectin. The latter is the most important polysaccharide in the cell wall of fruits and vegetables (Esquerré-Tugayé et al., 2000). Noted that, the pectinolytic activity of these microorganisms is the most studied among PCWDE, because it induced degradation of this protective wall, and softening of tissues (Selmaoui et al., 2017), which, led opportunistic plant pathogens that possess PCWDE enzymes to attack fruits and vegetables (the remaining 55 strains), and do not possess pectinase (Willats et al., 2001). Therefore, pectinase is the most important virulence factor of most pathogenic isolated microorganisms.

On this basis, these microorganisms can use a wide range of substrate to grow, making them more competitive and good colonizers. In 
addition to these enzymes, we also looked at their hemolytic activity. It is true that these germs contaminate the plant in the first place (no blood) but can pass to the human being, and cause diseases, most often serious after eating the infected fruits and vegetables (Linares et al., 2007) hence searching for hemolytic activity.

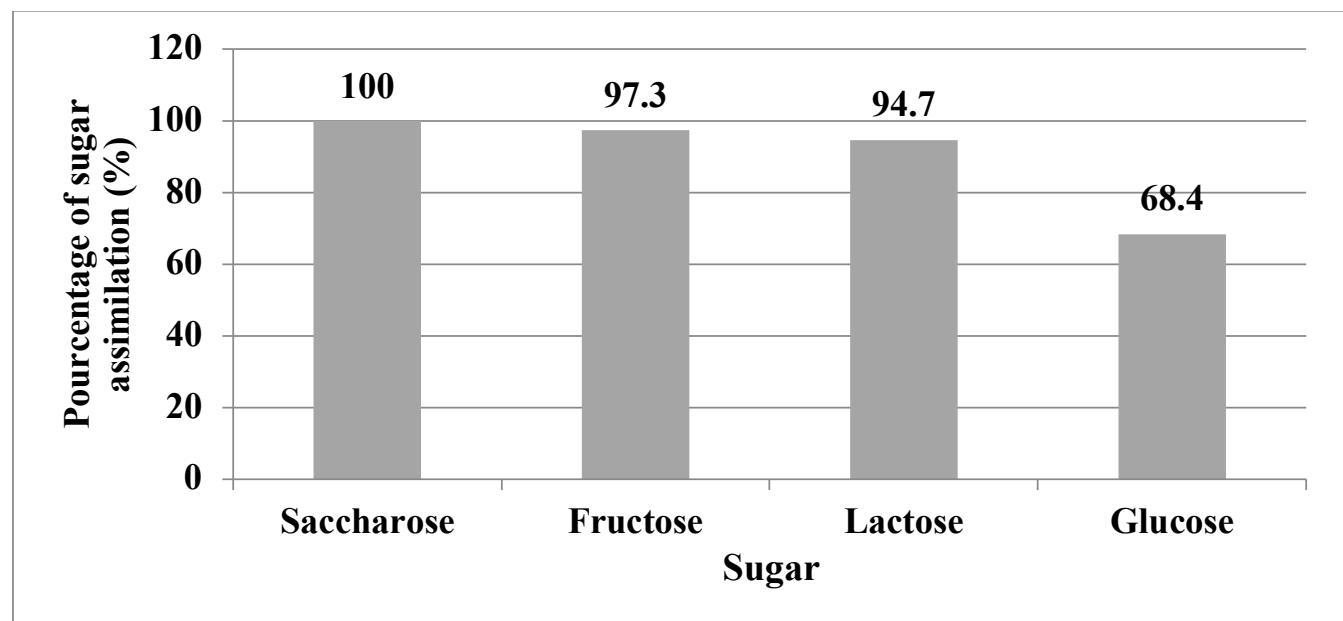

Figure 5. Histogram representing the percentage (\%) of sugar uptake by isolated microorganisms.

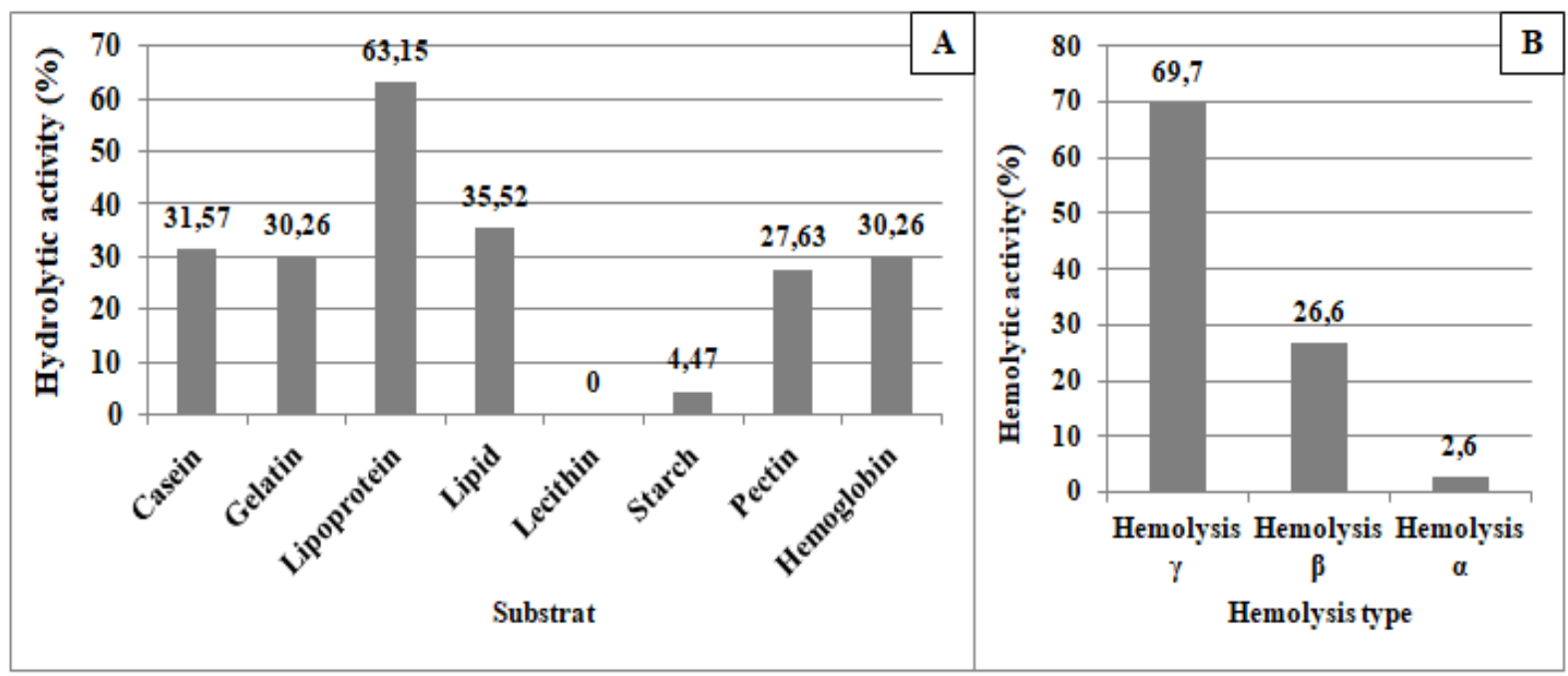

Figure 6. Histogram of hydrolytic activity of all strains involved in soft rot (A- hydrolytic activity by substrate type, B- hemolysis type by percentage of hemolytic activity).

\subsection{Identification of isolated} microorganisms

Based on the results presented in Tables 1 and 2, we observed that the isolated species belong mainly to the following six (06) genera in the three phylums:

- Yeasts: Candida and Trichosporon; - Fungi: Cladosporium and Botrytis;
- Bacteria: Staphylococcus and Neisseria.

Indeed, most of isolated and identified microorganisms belong to the fungi kingdom. Yeasts represent the dominant species in the division of Ascomycota, with the species Candida kefyr and Trichosporon sp. Consistent with our findings, Desbordes (2003) found that 
the genus Trichosporon is among pectinolytic yeasts that infect mostly vegetables. On the other hand, Rawat (2015) also found that Candida and its related genera cause human infections, but there is no literature that classifies Candida kefyr as a plant pathogen. This led us to assume that this species is a contaminant, because it known to be a human pathogen (Sanchis et al., 2016). These yeasts have been more frequently isolated from tomato, tangerine and apple. Second, the most abundant Fungi are represented by the species Cladosporium $s p$ isolated from mandarins and apples, followed by Botrytis $s p$, isolated mainly from onions. Finally, we note the two genera Fusarium and Penicillium, contrary to the studies of Abdullah et al. (2016) and Selmaoui et al. (2017).

This study also revealed the presence of bacteria associated with soft rot disease, the most dominant of which is the genus Neisseria. Suprinyan et al. (2012) have also isolated this genus from rotten fruit waste destined for biogas production. In addition, the species Staphylococcus xylosus isolated from strawberries and tomatoes is the second most common bacterial species; compared to Park et al. (2019).

However, we also found species such as Photobacterium damselae and Vibrio vulnificus, generally pathogens of raw seafood (Rivas et al., 2013; Copin et al., 2015). Their presence in our rotten fruits and vegetables is explained by contact with other seafood infected with these pathogens, or contamination by washing water. It is remembered that these identifications are relatively brief and certainly require a molecular study that may reveal other more likely species.

Indeed, yeast and Fungi have been found to infect fruit much more because they are rich in nutrients and water (Moss, 2008). In addition, the variation of the $\mathrm{pH}$ spectrum in fruits and vegetables, presents a competitive factor to promote the growth and infection of the latter by yeasts and Fungi, but not by bacteria (Warnasuriya et al., 1985). Because low $\mathrm{pH}$ values (2.2 to 5) prevent or delay the growth of bacteria, yeast and mould are able to grow in these $\mathrm{pH}$ ranges (Desbordes, 2003).

Furthermore, some species infect only one type of fruit or vegetable, such as Candida parapsilosis that has been isolated from the eggplant, and Staphylococcus xylosus isolated primarily from strawberries. In addition, other species are found in several fruits and vegetables, as is the case with the two (02) yeasts: Candida kefyr and Trichoporon sp.

\subsection{Pathogeicity test}

After 24 hours of incubation of the pectinolytic strains sown on the three (03) samples (zucchini, tangerine, tomato), we observed that the most samples showed symptoms of soft rot (Table 3). Moreover, pectinolytic strains PS2 (Trichosporon $s p$ ), PS9 (Staphylococcus xylosus), PS13 (Trichosporon $s p$ ) and PS19 (Geotrichum capitatum) caused harsh soft rot.

\subsection{Antimicrobial activity of common liquid disinfectants}

In the last part of this work, we agreed to test some tools for controlling the spread of these pectinolytic plant pathogens, using invitro test by three antimicrobial agents. The antimicrobial test is based on the spread of traditionally antimicrobial agents used for cleaning and disinfecting vegetables and fruits in households and food industries (bleach, white vinegar, sodium bicarbonate).

Based on the results obtained (Table 4), we noted that, depending on the diameter of the inhibition zone, the most effective disinfectant is vinegar, then bicarbonate, which gave a very significant antimicrobial activity, except for the bacterium PS10: Staphylococcus xylosus. The analysis of variance (Annex Table 1, 2 and 3) 
indicated a non-significant difference for disinfectants activities $(\mathrm{P}>0.05)$.

However, for diluted bleach, almost no inhibition zones were found, even at double and triple concentrations, meaning that pectinolytic strains are resistant to this disinfectant. Consistent with our findings, Fong et al. (2011) found that acetic acid and low $\mathrm{pH}$ of vinegar inhibits the growth of many pathogens, while sodium bicarbonate inhibits fungi growth with a very limited spectrum, probably because its alkalinity is not enough to eliminate all germs present on fruits and vegetables.

However, bleach activity was almost negligible, contrary to FDA expected results, even after doubling and tripling the concentration. This makes us think of two probabilities: the first is that pectinolytic microorganisms have developed resistance to chlorine compounds, due to the massive use of pesticides in the culture phase (Gava et al., 2018). The second probability is that the concentration used is too low to have a total inhibition of pectinolytic microorganisms (WHO, 2007).

\section{Conclusions}

In the light of the results obtained, it appears that of the 76 samples of rotten fruits and vegetables marketed in the town of Tamanghasset: 49 yeasts, 14 Fungi and 13 bacteria have been isolated and characterized. The most abundant species were Candida kefyr (tomato, mandarin); Cladosporium $s p$ (mandarin, apple); Botytis sp (onion); Staphylococcus xylosus (strawberry) and Neisseria cinerae (mandarin).

The results of the phenotypic tests carried out on the microorganisms in question have shown that soft rot is mainly of fungal origin, but this does not exclude the presence of bacteria. Isolated pathogens have a very diverse enzymatic equipment, whose key enzyme in phytopathology is pectinase, which has a role in the pathogenicity of our strains.
Furthermore, the production of other enzymes that are not necessarily involved in phytopathology, such as: hemolysin and lipoproteinase, by most of isolates identified, are an attractive results that can confirm the opportunism of these non-pectinolytic pathogens and their involvement in this disease. Therefore, it is mandatory to go towards preventive actions, by the use of common disinfectants, of which the most profitable in our study is white vinegar.

\section{References}

Abdullah, Q., Mahmoud, A., Al-Harethi, A. (2016). Isolation and identification of fungal post- harvest rot of some fruits in Yemen. PSM Microbiology, 1(1), 36 - 44.

Alfano, J. R., Collmer, A. (1996). Bacterial pathogens in plants: life up against the wall. Plant Cell 8:1683-1698., Jay, J. M. 1996. Modern food microbiology, 5th ed., pp408424. Chapman \& Hall, London.

Amin, L. A., Yapi, P. D. Y. A., Monnet, Y. T., Yapi, M. A. Y., Soro, C. L., Kouadio, K. K. (2016). Evaluation microbiologique et origines de la contamination des produits de

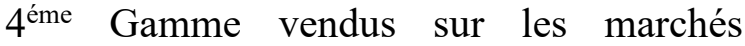
d'Abidjan, Cote d'ivoire. European scientific Journal, 1236, 273 - 285.

Aouar, L. (2012). Isolement et identification des actinomycètes antagonistes des microorganismes phytopathogénes. Thèse de Doctorat en Sciences. Doctoral dissertation. University MentouriConstantine, Algeria, p34.

Benidire, L. Daouik Fatemi Z. A., Achouak, W., Bouarah, L., Oufdou, K. (2015). Effet de stress salin sur la germination et le développement des plantules de Vicia faba $L$ (Effet of salt stress on germination and seedling of Vicia faba L. Journal of merterials and environnemental science, 6(3), $840-851$.

Boiron, P. (1996). Organisation et biologie des champignons. p19-79, Ed Nathan, Paris, France. 
Castro-Escapulli, G., Figueras, M. J., AguileraArreola, G., Soler L., Fernadez-Rendom, E., Aâricio, G. O.,... Chacon, M. R. (2003). Characterization of Aeromonas spp: isolated from frozen fish intended from human consumption in Mexico. International Journal of Food Microbiology, 84(1), 41-49

Copin, S., Annick, R. P., Mélanie, G., Quilici, M. L. (2015). Vibrio impliqués en pathologie humaine : une étude de leur répartition dans des produits de la mer consommés en France. Bulletin épidémiologique, santé animale et alimentation/Spécial Vigilance sur la chaîne alimentaire, 68, 21-25

Delarras, C. (2007). Microbiologie pratique pour le laboratoire d'analyses ou de contrôle sanitaire. Edition Tec \& Doc, Lavoisier, Paris, France.

Delarras, C. (2014). Pratique en microbiologie de laboratoire : recherche de bactéries et de levure-moisissures. Edition Tec \& Doc, Lavoisier, Paris, France.

Desbordes, D. (2003). Qualité microbiologique des fruits et légumes: flores, altération, risques sanitaires, prévention. p19 -24, Rapport de recherche bibliographique, Lyon.

Dolisi, G. (2007). Saccharomyces cerevisaie : observation, coloration, mise en culture. Bio-Top.

Dufresne, P., Guy, S. G. (2018). Identification des champignons d'importance médicale. Laboratoire de santé publique du Québec. p5-9, Institut National de Santé Publique. Canada.

Egamberdiyeva, D., Höflich G. (2004). Effet of Plant Growth Promoting Bacteria on growth and nutrient uptake of cotton and pea in a semi-arid region of Uzbekistan. Journal of Arid Environnents, 56(2), 293301.

Esquerré-Tugayé, M. T., Boudart, G., Dunas, B. (2000). Cell Wall Degrading Enzymes, inhibitory proteins, and oligosaccharides participate in the molecular dialogue between plant and pathologens. Plant Physiology and Biochemistry, 38(1/2), 157163

Fong, D., Gaulin, C., Lê, M. L., Shum, M. (2011). Efficacité des agents antimicrobiens de substitution pour la désinfection des surfaces dures. Centre de collaboration nationale en santé environnementale, 1-22.

Gava, C. A. T., de Castro, A. P. C., Pereira, C. A., Fernandes-Júnior, P. I. (2018). Isolation of fruit colonizer yeasts and screening against mango decay caused by multiple pathogens. Biological Control, 117, 137146.

Gram, L., Ravn, L., Rasch, M., Bruhn, J. B., Christensen, A. B., Givskov, M. (2002). Food spoilage-interactions between food spoilage bacteria. International journal of food microbiology, 78(1-2), 79-97.

Gupta, V. K., March, R. L., Screenivasaprasad, S. (2015). Fungal Biomolecules: Sources, Applications and Recent Developments. $p$ 291, Ed John Wiley \& Sons. Pondicherry, India.

Hoeffler, U. (1977). Enzymatic and hemolytic properties of Propionibacterium acnes and related bacteria. Journal of clinical microbiology, 6(6), 555-558.

Hozbor, M. C., Saiz, A. I., Yeannes, M. I., Fritz, R. (2006). Microbiological changes and its correlation with quality indices during aerobic iced storage of sea salamon (Pseudopercis semifasciata). LWT-Food Science Technology, 39(2), 99-104.

Ife Fitz, J., Bas, K. (2003). La conservation des fruits et légumes. pp12, Editor: Bas kulpers. Fondation Agromisa, Wageningen, Pays Bas.

Lee, D. H., Kin, J. B., Kin, M., Roh, E., Jung, K. (2013). Microbiota on spoiled. Vegetables and their characterization Journal of food protection, 76 (08), 13501358.

Linares, C. E., Loreto, E. S. D., Silveria, C. P., Pozzatti, P., Scheid, L.A., Santurio, J. M., Alves, S. H. (2007). Enzymatic and hemolytic activities of Candida 
dubliniensis strains . Renista do instituto de Medicino tropical de Sao Paulo, 49(4), 203-206.

Lopez Camelo, A. F. (2007). Manual for the preparation and sale of fruits and vegetables. From the field to the market. Food and Agriculture Organization Agricultural Services Bulletin (FAO), p82.

Miyadoh, S. (1993). Research on antibiotic screening in Japan over the last decade: a producing microorganism approach. Actinomycetes, 7(2), 100-106.

Moss, M. O. (2008). Fungi, quality and safety issues in fresh fruits and vegetables. Journal of Applied Microbiology, 104(5), 1239-1243.

Movahedi, S., Heale, J. B. (1990). Purification and characterization of an aspartic proteinase secreted by Botrytis cinerea Pers ex. Pers in culture and in infected carrots, Physiological and Molecular Plant Pathology, 36(4), 289-302.

Nicklin, J., Graeme-Cook, K., Paget T., Killington R. (2000). L'essentiel en microbiologie. Ed Berti. Paris, France, pp 210-216.

Park, K. M., Kim, H. J., Kim, S. S., Lee, S. B., Jeong, M., Park, K. J., Koo, M. (2019). Effect of temperature treatment on postharvest quality of the cherry tomato (Lycopersicon esculentum var. cerasiforme). Korean Food Conservation Journal , 26(6), 595-605. DOI : $10.11002 / \mathrm{kjfp} .2019 .26 .6 .595$

Perombelen, M. C. M. (2002). Potato diseases caused by soft rot Erwinia: an overview of pathogenesis. Plant pathology, 51(1), 1-12.

Pozzi, A. C. (2014). Rôles adaptatifs et contraintes de la sporulation chez les microorganismes associés aux plantes: cas de la sporulation in planta dans la symbiose actinorhizienne Frankia (Frankiaceae)Alnus (Betulaceae) (Doctoral dissertation). Université Claude Bernard, France, p 359.

Rawat, S. (2015). Food spoilage : Microorganisms and their prevention. Asian journal of plant science and Research, 5(4), $47-56$.

Rémésy, C. (2008). "Sucre simple purifiés versus sucres des fruits, ont-ils les mêmes effets métabolique ? ». Phytothérapie, 6(2), 91-95. https://doi.org/10.1007/s10298-0080297-z

Rivas, A. J., Lemos, M. L., Osorio, C. R. (2013). Photobacterium damselae subsp. damselae, a bacterium pathogenic for marine animals and humans. Frontiers in Microbiology, 4(283), 1-6.

Rosset, R. (1990). Légumes de 4 et $5^{\mathrm{e}}$ gamme microbiologie et toxi-infections alimentaire collectives. Bulletin de l'Académie Vétérinaire de France, 63(3), 43-55.

Sanchez, M. C. (2018). Food law and regulation for non-lawyers: a US perspective. Springer.

Selmaoui, K., Touhami, A. O., Mouria, A., Benkirane, R., Douira, A. (2017). Détection de l'activité enzymatique pectinolytique et cellulolytique $\mathrm{du}$ champignon responsable de la pourriture des pommes en conservation. International journal of immovation and scientific research, 30(2), 242-250.

Snaiki, J., Nadif, A., Ouhssine, M. (2006). Détéction biochimique d'Erwinia carotovora susbsp carotovora de tubercules de bettrave sucrière atteints de pourriture molle. Bulletin-Société de Pharmacie de Bordeaux, 145(1/4), 53-60

Suprinyan, A., Rohimah, I., Suryani, Y., Sa'adah, S. (2012). Isolation and identification of cellulolytic bacteria from waste organic vegetables and fruits for role in making materials biogas. Journal ISTEK, 6(1-2), 10-20.

Toth, I. K., Bell, K. S., Holeva, M. C., Birch, P. R. J. (2003). Soft rot Erwinia: from genes to genomes. Molecular Plant Pathology, 4, $17-30$.

Wallen, S. E. (1983). Understinding Botulisin. Dairy and food sanitation, 3(1), 4-8.

Warnasuriya, D., Liyanage, A., Weerawansa G. G., Athauda P.K., Jayatissa. (1985). 
Isolation and characterization of yeasts of some fruits and fruit products of Srilanka. Journal of national science Council Srilanka, 13(2), 1-7

World Health Organization [WHO] (2007). Preventing and combating acute epidermal or pandemic respiratory diseases as part of care. $\mathrm{p} 81$.

Zalewsky-Sobcazak, J. (1985). Sequential secretion of Cell Wall Degrading Enzymes by Botrytis fabae and Fusarium avenaceum during Growth on Host and Non-Host Plants. Biochemie und Physiologie der Pflanzae, 180(3), 169-175.

\section{Annex : Analysis of variance}

Table A1. Bicarbonate's activity

\begin{tabular}{|l|r|r|l|l|l|}
\hline Source & DDL & Sum of squares & $\begin{array}{l}\text { Average of } \\
\text { squares }\end{array}$ & F & $\operatorname{Pr}>$ F \\
\hline Model & 20 & 447.524 & 22.376 & 6.293 & $\mathbf{0 , 0 0 0 1}$ \\
\hline Error & 42 & 149.333 & 3.556 & & \\
\hline Total corrected & 62 & 596.857 & & & \\
\hline
\end{tabular}

Table A2. Bleach's activity

\begin{tabular}{|l|r|r|l|l|l|}
\hline Source & DDL & Sum of squares & $\begin{array}{l}\text { Average of } \\
\text { squares }\end{array}$ & F & $\operatorname{Pr}>$ F \\
\hline Model & 20 & 444.317 & 56.683 & 27.260 & $\mathbf{0 , 0 0 0 1}$ \\
\hline Error & 42 & 84 & 2 & & \\
\hline Total corrected & 62 & 528.317 & & & \\
\hline
\end{tabular}

Table A3. White vinager's activity

\begin{tabular}{|l|r|r|l|l|l|}
\hline Source & DDL & $\begin{array}{l}\text { Sum of } \\
\text { squares }\end{array}$ & $\begin{array}{l}\text { Average of } \\
\text { squares }\end{array}$ & F & $\operatorname{Pr}>$ F \\
\hline Model & 20 & 1133.651 & 27.260 & 27.260 & $<\mathbf{0 , 0 0 1}$ \\
\hline Error & 42 & 87.333 & & & \\
\hline Total corrected & 62 & 1220.984 & & & \\
\hline
\end{tabular}

